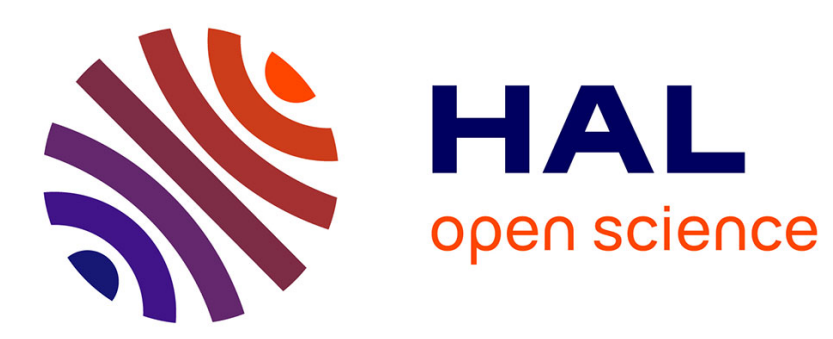

\title{
Augmentation de la hauteur de barrière de diodes de Schottky au silicium: Application aux cellules solaires
}

\author{
J.P. Ponpon, P. Siffert
}

\section{To cite this version:}

J.P. Ponpon, P. Siffert. Augmentation de la hauteur de barrière de diodes de Schottky au silicium: Application aux cellules solaires. Journal de Physique Lettres, 1975, 36 (5), pp.149-151. 10.1051/jphyslet:01975003605014900 . jpa-00231175

\section{HAL Id: jpa-00231175 https://hal.science/jpa-00231175}

Submitted on 1 Jan 1975

HAL is a multi-disciplinary open access archive for the deposit and dissemination of scientific research documents, whether they are published or not. The documents may come from teaching and research institutions in France or abroad, or from public or private research centers.
L'archive ouverte pluridisciplinaire HAL, est destinée au dépôt et à la diffusion de documents scientifiques de niveau recherche, publiés ou non, émanant des établissements d'enseignement et de recherche français ou étrangers, des laboratoires publics ou privés. 


\title{
AUGMENTATION DE LA HAUTEUR DE BARRIÈRE DE DIODES DE SCHOTTKY AU SILICIUM : APPLICATION AUX CELLULES SOLAIRES
}

\author{
J. P. PONPON et P. SIFFERT \\ Centre de Recherches Nucléaires \\ Laboratoire de Physique des Rayonnements \\ et d'Electronique Nucléaire, 67037 Strasbourg Cedex, France
}

(Reçu le 6 février 1975, accepté le 3 mars 1975)

\begin{abstract}
Résumé. - Le rendement des cellules solaires au silicium réalisées par diode Schottky dépend essentiellement de la hauteur de la barrière de potentiel au contact métal-semiconducteur. On propose ici deux procédés permettant de porter cette barrière aux environs de $1 \mathrm{eV}$, soit par évaporation de hafnium sur du silicium $\mathrm{P}$, soit par une implantation d'ions avant la formation d'une diode Schottky à l'or sur du silicium $\mathbf{N}$.
\end{abstract}

\begin{abstract}
The efficiency of Schottky silicon solar cells depends strongly on the barrier height of the metal-semiconductor contact. We show that it is possible to increase this barrier height up to about $1 \mathrm{eV}$ either by the use of hafnium contacts on $\mathrm{P}$ type silicon, or by making an ion implantation before the realisation of a gold Schottky diode on $\mathrm{N}$ type silicon.
\end{abstract}

Introduction. - Les cellules solaires au silicium, largement utilisées dans les expériences spatiales pour la conversion en électricité de l'énergie solaire, sont préparées par diffusion d'une impureté donatrice dans un substrat de type P. Leur rendement de conversion atteint actuellement $15 \%$ dans les meilleurs des cas (à savoir pour une profondeur de jonction de l'ordre de $0,2 \mu$ et des conditions de réalisation optimales pour ce qui concerne la grille collectrice et la couche antiréfléchissante [1, 2]) alors que la limite calculée est d'environ $23 \%$. L'application terrestre à grande échelle de ces cellules nécessite une réduction importante des coûts de production, d'un facteur 100 au moins. Les diodes Schottky représentent une alternative intéressante pour la réalisation de tels dispositifs du fait de leur plus faible prix de revient et de leur mince fenêtre d'entrée, qui permettrait une amélioration de la réponse de côté des courtes longueurs d'onde. Toutefois, peu de travaux ont été effectués dans ce domaine $[3,4,5]$ et les rendements atteignent à peine $8 \%$. Comme on le verra ci-après, ceux-ci sont limités par la faible hauteur de barrière $\Phi_{\mathrm{Bn}}$ au contact métal-semiconducteur. Nous montrons ici qu'il est possible de porter les valeurs de $\Phi_{\mathrm{Bn}}$ à $1 \mathrm{eV}$ et, par conséquent, d'augmenter considérablement l'efficacité de conversion.
1. Rendement de conversion des diodes Schottky au silicium. - Loferski [6] a montré que l'efficacité de conversion $\eta$ d'une cellule solaire, égale au rapport de la puissance délivrée par la cellule à la puissance photonique d'entrée $P$ par unité de surface est donnée par la relation :

$$
\eta=\frac{\mathrm{e} V_{\mathrm{m}}^{2} I_{0}}{k T P} \exp \left(\frac{\mathrm{e} V_{\mathrm{m}}}{k T}\right)
$$

pour une diode de Schottky

$$
I_{0}=A^{* *} T^{2} \exp \left(-\frac{\mathrm{e} \Phi_{\mathrm{Bn}}}{k T}\right)
$$

$A^{* *}$ étant la constante effective de Richardson et $V_{\mathrm{m}}$ représente la tension aux bornes de la cellule à la puissance maximale, calculée d'après l'expression :

$$
\left(1+\frac{\mathrm{e} V_{\mathrm{m}}}{k T}\right) \exp \left(\frac{\mathrm{e} V_{\mathrm{m}}}{k T}\right)=1+\frac{I_{\mathrm{p}}}{I_{0}}
$$

où $I_{\mathrm{p}}$ est le photocourant généré sous illumination.

Les autres symboles ont leur signification habituelle.

Nous avons calculé l'efficacité maximale $\eta$ pour un éclairement dans l'espace (AMO) en négligeant les pertes par réflexions et recombinaisons et en supposant le rendement quantique égal à 
l'unité. Dans ces conditions $P=0,135 \mathrm{~W} / \mathrm{cm}^{2}$; $I_{\mathrm{p}}=60 \mathrm{~mA} / \mathrm{cm}^{2} ; A^{* *}=96 \mathrm{~A} / \mathrm{cm}^{2} \mathrm{~K}^{2}$.

Les résultats de ces calculs sont reportés sur la figure 1 en fonction de la hauteur de barrière $\Phi_{\mathbf{B n}}^{*}$ pour différentes températures de fonctionnement. A température ambiante, on montre que :

$$
\eta(\%)=41,2 \Phi_{\mathrm{Bn}}(\mathrm{eV})-22,7 \text {. }
$$

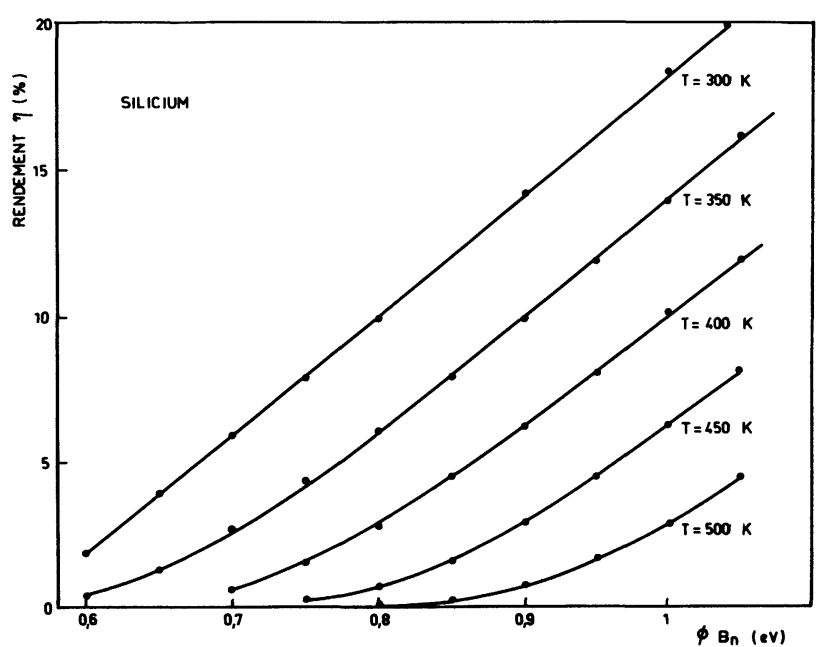

FIG. 1. - Evolution du rendement de conversion des cellules solaires du type diode Schottky en fonction de la hauteur de la barrière de potentiel, à différentes températures.

Généralement les valeurs de $\Phi_{\mathrm{Bn}}$ sont de l'ordre de $0,8 \mathrm{eV}$ quelle que soit la résistivité du matériau de base [7], dans une grande plage de température, ce qui limite les rendements à $10 \%$ environ. Une augmentation de $\Phi_{\mathrm{Bn}}$ à $1 \mathrm{eV}$ conduirait presque à doubler ce chiffre. Nous proposons deux méthodes pour atteindre ce but.

2. Barrière hafnium-silicium $\mathbf{P}$. - Nous avons montré récemment que la hafnium déposé sur le silicium de type $P$, donne une hauteur de barrière $\Phi_{\mathrm{Bn}}$ égale à $0,95 \mathrm{eV}$ à température ambiante, sans formation de siliciure. Ces résultats, ayant fait l'objet d'une publication récente [8], ne seront pas développés ici.

3. Barrière or-silicium $N$ prébombardé. - La présence d'une zone superficielle de très faible épaisseur et de type de conduction opposé à celui du substrat peut provoquer, dans certaines conditions, une augmentation notable de la hauteur de la barrière de potentiel d'une diode Schottky, du fait de la modification de la courbure des bandes. Une telle zone peut être produite par implantation ionique, processus qui permet un contrôle aisé de la densité d'impuretés introduites et de leur profondeur de pénétration. Dans du silicium de type $\mathrm{N}$, l'introduction d'ions accepteurs créé une zone de charge d'espace négative qui augmente le potentiel à la surface pour les électrons, à condition que la dose implantée soit supérieure à une valeur minimale $D_{0}$ telle que :

$$
D_{0}=\left[\frac{2 \varepsilon V_{0}}{\mathrm{e}}\left(N_{\mathrm{D}}-N_{\mathrm{A}}\right)\right]^{1 / 2}
$$

où $\left(N_{\mathrm{D}}-N_{\mathrm{A}}\right)$ représente la densité d'impuretés ionisées du substrat $N$ et $V_{0}$ la courbure des bandes de la barrière de Schottky conventionnelle. La hauteur de barrière $\Phi_{\mathrm{Bn}}$ augmente avec la dose tant que la zone implantée demeure totalement désertée en l'absence de toute polarisation extérieure. Au-delà il y a formation d'une jonction P-N.

L'utilisation de ce procédé a permis à Shannon [9] de porter la hauteur de barrière de diodes nickelsilicium $\mathrm{P}$ de 0,49 à $0,75 \mathrm{eV}$ en implantant des ions antimoine de $5 \mathrm{keV}$. Nous avons étudié des diodes or-silicium $\mathrm{N}$, pour lesquelles la hauteur de barrière était initialement $0,8 \mathrm{eV}$ et avons utilisé des ions gallium de $30 \mathrm{keV}$ dans du silicium contenant en moyenne $5,5 \times 10^{14} / \mathrm{cm}^{3}$ impuretés ionisées. La courbure des bandes $V_{0}$ étant égale à $0,5 \mathrm{~V}$ la valeur de $D_{0}$ est de l'ordre de $6 \times 10^{11} / \mathrm{cm}^{2}$; nous avons par conséquent introduit des doses comprises entre $10^{12}$ et $2,5 \times 10^{13} / \mathrm{cm}^{2}$ et procédé à un recuit des échantillons à $400^{\circ} \mathrm{C}$ afin de rendre une partie des ions implantés électriquement actifs. Les hauteurs de barrière ont été déterminées par une mesure de la capacité $C$ des diodes en fonction de la tension de polarisation inverse. L'extrapolation des droites $S^{2} / C^{2}$ (où $S$ est la surface des diodes) donne la valeur $V_{S}$ de la courbure des bandes; $\Phi_{\mathrm{Bn}}$ se calcule alors par la relation :

$$
\Phi_{\mathrm{Bn}}=V_{S}+\frac{k T}{\mathrm{e}} \ln \left(\frac{N_{\mathrm{c}}}{N_{\mathrm{D}}-N_{\mathrm{A}}}\right)
$$

où $\mathrm{N}_{\mathrm{c}}$ est le nombre d'états dans la bande de conduction. Pour des doses implantées de $10^{12}, 5 \times 10^{12}$ et $10^{13}$ ions $\mathrm{Ga}^{+} / \mathrm{cm}^{2}$ les valeurs de $\Phi_{\mathrm{Bn}}$ trouvées sont respectivement égales à $0,91,0,96$ et $0,99 \pm 0,02 \mathrm{eV}$. Une dose de $2,5 \times 10^{13} / \mathrm{cm}^{2}$ provoque la formation d'une jonction P-N.

On peut noter que la capacité des diodes varie avec la fréquence du signal de mesure délivré par le capacimètre, ce qui indique la présence de niveaux d'énergie profonds situés dans la bande interdite et produits lors du bombardement. Une étude plus

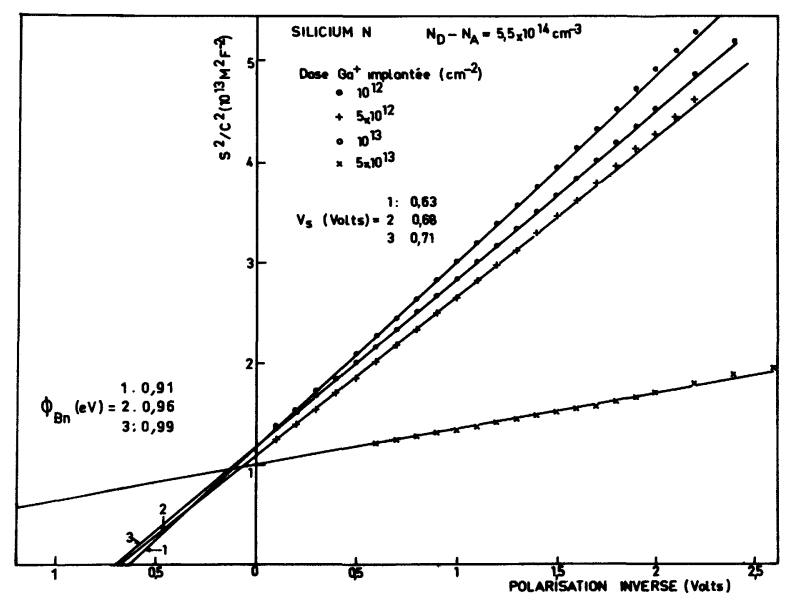

FiG. 2. - Détermination, par mesure de la capacité inverse, de la courbure des bandes Vs de diodes Schottky à l'or prébombardées par des ions gallium. 
détaillée des conditions du recuit devrait permettre d'éliminer ces derniers. On peut également envisager l'implantation d'ions plus légers (bore) qui créent moins de dommages. Toutefois, afin de conserver une zone de très faible épaisseur (environ $100 \AA$ ), il est nécessaire de réduire l'énergie de ces derniers à quelques $\mathrm{keV}$.

4. Conclusion. - Nous avons montré que plusieurs procédés conduisant à une barrière de potentiel de l'ordre de $1 \mathrm{eV}$ peuvent être utilisés sur le silicium, ce qui devrait permettre d'augmenter de façon notable l'efficacité de ces dispositifs. De nombreux points restent cependant à étudier, en particulier l'épaisseur des contacts métalliques, leur mode de réalisation (évaporation ou pulvérisation cathodique), les conditions optimales d'implantation et de recuit des échantillons après le bombardement ionique.

\section{Bibliographie}

[1] Lindmayer, J. et Allison, J., 9 IEEE Photovoltaic Specialists Conference (Silver Springs) 1972 p. 83.

[2] Amingual, D., Colardelle, P. et Nguyen Duy, T., Int. Conf. on Photovoltaic Power Generation, Hambourg 1974 p. 85 ;

RalPh, E. L., ScotT-MoncK, J., Int. Conf. on Photovoltaic Power Generation, Hambourg 1974 p. 103.

[3] Ahlstrom, E., J. Appl. Phys. 33 (1962) 2602.

[4] Anderson, W. A., Delahoy, A. E. et Milano, R. A., J. Appl. Phys. 45 (1974) 3913.
[5] Pulfrey, D. L. et Mc Ouat, R. F., Appl. Phys. Lett. 24 (1974) 167.

[6] LOFERSKI, J. J., J. Appl. Phys. 27 (1965) 777.

[7] Chang, C. Y., Sze, S. M., Solid State Electron. 13 (1970) 727.

[8] Saxena, A. N., Grob, J. J., Hage-Ali, M., Siffert, P., Metal Semiconductor Contacts Conf. Series $\mathrm{n}^{\circ} 22$, the Institute of Physics London (1974).

[9] Shannon, J. M., Appl. Phys. Lett. 25 (1974) 75. 